\title{
Cortical Software Re-Use: A Computational Principle for Cognitive Development in Robots
}

\author{
Ronan G. Reilly \\ Department of Computer Science \\ National University of Ireland, Maynooth \\ Co. Kildare, Ireland \\ Ronan.Reilly@may.ie
}

\author{
Ioana D. Marian \\ Department of Computer Science \\ University College Dublin \\ Belfield, Dublin 4, Ireland \\ Ioana.Marian@ucd.ie
}

\begin{abstract}
The goal of this paper is to propose a candidate for consideration as a computational principle for cognitive development in autonomous robots. The candidate in question is the theory of Cortical Software Re-Use (CSRU) and we will make the case in this paper that it provides a mechanism for the incremental construction of cognitive and language systems from simpler sensory-motor components.
\end{abstract}

\section{Introduction}

There is a growing view among robotics researchers that to be truly intelligent, robots need to be capable of autonomous mental development $[9,17,20]$. If the goal is to build complete, integrated systems that can operate at human levels of performance, we must find appropriate methodologies that allow us to scale both the size and complexity of their behavioral repertoires [17]. One obvious source of inspiration is the process of human cognitive development. Among the attractive features of a developmental approach are its open-endedness, its biological plausibility, and the incrementally increasing behavioral complexity in a principled way. There is, we believe, a gradual trend in robotics research away from off-line, pre-programmed system design and construction to a more epigenetic approach. The hope is that within this paradigm, increasingly more complex cognitive structures will emerge in these systems as a result of interactions with the physical and social environment [22].

Much work to date on implementing a developmental methodology for robots has been derived from the classical paradigms of developmental psychology $[6,7,17]$. The terms of description are behavioral and cognitive: developmental stages, accommodation and assimilation of behavior, and so on. The current focus of much of this research is on finding a way to specify these concepts at a neural and implementational level. For example, one group of robotics researchers has taken the skills acquired in learning to foveate a target and re-used them in the execution of reaching and head movements $[9,17]$. Another strand of research has explored how intentionality emerges from a process of learning goal-method associations [6], and how communication develops on the basis of joint attention involving gaze and deixis [6, 17]. However, all of these efforts are quite task specific, and cannot be used as a general developmental framework. What is needed, therefore, is the specification of a general, implementable developmental methodology capable of supporting the gradual development of higher cognitive functions from simpler components. An appropriate developmental methodology should fulfill the following criteria:

- provide a mechanism for constructing more complex behaviors from simpler components;

- be specified at behavioral, computational, and neural levels;

- be capable of accounting for the emergence of higher level cognitive functions (i.e. cognition, planning, language).

We propose a candidate framework in the form of Cortical Software Re-Use (CSRU) theory.

\section{CSRU principles}

The central concept of the theory, that of "software re-use", is borrowed from the field of software engineering. Put simply, it states that dynamical neural processes from the sensory-motor areas of the brain provide the computational building blocks for higher 
level functions up to and including those involved in cognition and language.

The principles of CSRU were first elaborated by Reilly [13] to account for syntax acquisition in terms of the re-use of a motor program for complex action sequences. They are:

- Cortical "algorithms" for language processing and cognition are derived from, and built upon, those from the sensory-motor domain. According to this view, cortical circuits that are involved in the planning of motor movements, say, can be exploited during reasoning, and not necessarily when reasoning about movement. This process is the basis for the simplest form of cortical re-use. An example of how this might work is given in section 3.1.

- The functioning of these algorithms is mediated by reciprocal projections between sensory, motor, association, and prefrontal areas. The direction of influence is from the sensory and motor regions to the higher cortical regions, because the circuitry in the sensory-motor areas consolidates and matures earlier, and that of the prefrontal area remains plastic the longest.

- The style of computation is a form of dynamical constraint satisfaction, where patterns of neuronal firing from connected regions mutually influence one another through a process of resonance and harmonization. In computational terms one can think of two resonant dynamical patterns as being equivalent to the application of a function designed for one domain (e.g., sensory-motor) to a new domain (e.g., cognitive). The last two principles lay the foundation for a complex form of dynamical re-use, referred as asymmetric cell assemblies collaboration (section 3.2).

If we are to build neurally inspired complex artificial systems, it is essential to identify the fundamental computational building blocks. Such a basic component is likely to be intrinsic, emerging early in development, and not requiring the intervention of learning, at least in its initial form. The neural primitive proposed by CSRU is the collaborative cell assembly (CCA). Here, software re-use indicates that the developing partner in a collaboration is able to re-use the repertoire of cell assemblies already established in the more developed cortical regions. What is new about this proposal is that it focuses on the interaction between these types of cell assembly and the possible role that development may play in this interaction. CSRU makes a distinction between collaborations involving cell assemblies that are equally well developed, and those in which one partner in the collaboration is more developed than the other. We refer to the former as symmetric collaboration and the latter as asymmetric. In the later case, there is the possibility for the less well developed cell assembly to exploit the functionality of the more developed one. We illustrate in the following sections how these computations may take place.

\section{CSRU developmental methodology}

We have proposed above three criteria that a developmental methodology should fulfill if it aims to be applied to cognitive development in robots. We believe that such a methodology can and should be grounded in a psychological account of human development, be neurally plausible, and computationally implementable. For example, the concepts underlying CSRU have been around in various guises for some time. The notion of re-use is very much in harmony with adaptation-driven design principles found in both evolution and development [10]. Cell assemblies are envisaged along the lines proposed by Hebb and later Pulvermüller [4, 12]. What is new about CSRU and what makes it a feasible developmental methodology, is that it describes development in relatively explicit neural terms. We propose below, two forms of re-use, which, we maintain, give us the computational means ultimately for development of cognitive capabilities.

\subsection{Neighborhood collaboration}

A key issue for CSRU is how relevant perceptuomotor functions are selected or "indexed" for re-use by higher-level functions. CSRU assumes the indexing to be domain or content independent, relying on the relatedness of neuronal firing patterns at an abstract structural level. Therefore, there need not necessarily be a "semantic" connection between the re-used component and its new application. An illustrative example is the proposal by Greenfield [3] regarding the dual function of Broca's area. She observed parallels in the developmental complexity of speech and object manipulation of children aged 11-36 months. Using evidence from neurology, neuropsychology, child development, and animal studies, she argued that the two processes are built upon an initially common neurological foundation, which then divides into separate specialized areas as development progresses.

CSRU theory [13] was used to provide a simulation account of how the motor programs developed for object manipulation might be re-used for language syntax. In CSRU terms, the object assembly special- 


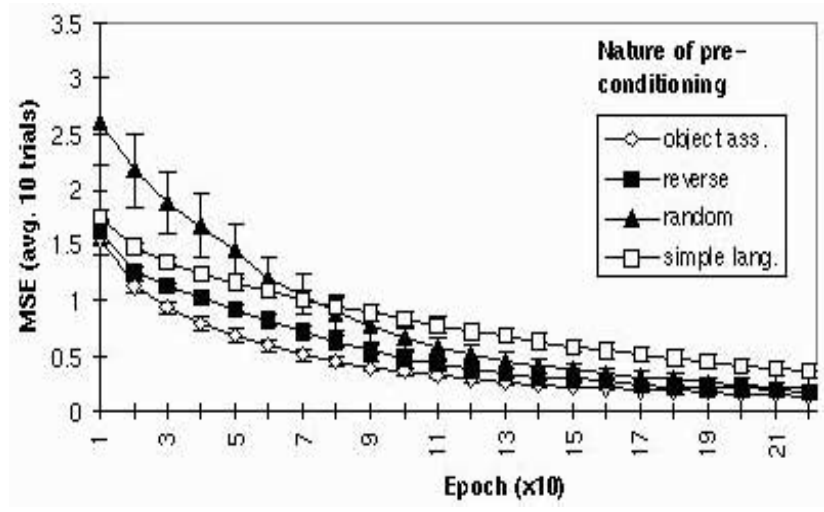

Figure 1: The relative effects on performance of learning a language production task with a simple recurrent network, when different pretraining regimes are applied.

ization furnishes the necessary re-usable primitives for the construction of language production system. The question of re-usability was operationalized by looking for a training advantage when a simple recurrent network (SRN) was pre-trained on an object assembly task prior to learning a language task. A number of control conditions were implemented: random initialization of the language network, pre-training the network on input with similar numerical properties to the object assembly task, pre-training the network with the language production task first.

Figure 1 shows the average performance of several SRN networks after the introduction of a simple language corpus, and as a function of different preconditioning regimes. The bottom line shows that pretraining on an object assembly task is more advantageous than the other control conditions in facilitating the emergence of a simple syntax capability.

In the case of Greenfield's proposal, re-use actually involves exploiting more or less the same cortical region. The selection of re-usable functions from one domain for re-use in another is based, at least in part, on structural isomorphism, possibly supported by resonance between the firing patterns of the re-using and re-used cortical regions. Topographic proximity is also obviously a factor. Therefore, in Greenfield's case, at some level of abstraction there is an isomorphism between the neuronal activity underlying motor sequence planning and speech planning. The child's emerging speech capability indexes relevant functions of the motor planning system by virtue of this structural isomorphism, and exploits them during development. This is re-use in its simplest form. Recent work on the mathematical foundations of the CSRU theory have established the basis of a more complex form of reuse: collaborative cell assemblies [14].

\subsection{Asymmetric collaboration}

A key challenge to implementing a developmental methodology is translating behavioral level accounts into neurocomputational ones. CSRU helps bridge the gap by using a mathematical framework that in the last decade has been increasingly exploited as a means of understanding brain function both at a neural and cognitive level. A dynamical systems theory account of cognition sees cognitive processes as behavioral patterns of non-linear dynamical systems [11]. Previous work in our group has focused on developing a simulation environment for networks of spiking neurons [8], which now allows us to explore properties of dynamical neural systems which are essential to understanding CSRU computations, such as: synchronization of firing patterns, and selection of re-usable modules on the base of resonance and structural isomorphism.

Asymmetric collaboration is based on a computational mechanism, by which neuronal units are capable not only of learning and reproducing a pattern but, more importantly, of creating new types of behavior by superimposing (or applying some other type of linear or nonlinear combining rule) patterns it is exposed to. More specifically, the periodic behavior displayed by one or more neural assemblies from the relatively well-developed area (a motor area in our case) is used as an input for a network in a developing area (cognitive function areas) and by adapting the synaptic weights of the "student network" the oscillator is capable of compounding the inputs into a more complex pattern. Current work is underway to implement a small-scale simulation of the dynamics in two neural populations, as an example of asymmetrically collaborating cell assemblies. Our specific focus is on motor sequence learning and reproduction (see section 5).

We have argued so far that CSRU can provide a neural-level account for aspects of the development of syntax production by using extant motor programs. Furthermore, we propose that the collaborative cell assemblies framework can support the development of deferred imitation and intentional search in robots. Prior to introducing our view of these developmental processes, we discuss a critical issue in building robots that imitate.

\section{Visuo-Motor mapping from re-use perspective}

An essential problem in learning by imitation is how to map an observed action to the appropriate motor commands $[1,7]$. While the CSRU paradigm proposes 
that one can develop complex cognitive capabilities from a repertoire of sensory-motor programs, there is still the issue of how this basic repertoire of sensorymotor programs (e.g., eye-hand coordination) is acquired. In the following, we present our approach to modeling a learning process for the acquisition of a basic imitative skill.

Results from neurophysiological studies of the visual analysis of motion have established the existence of a special pathway for processing the direction of movement (i.e., the dorsal pathway) [5]. Furthermore, it has been shown that at all stages of motor control, the activity of a substantial percentage of movementrelated neurons depends upon the direction of movement (i.e., involve direction selective cells) [5].

Our idea was to consider the activity of the motionselective cells as a low-level neural code, which is uniformly present throughout the brain areas involved in processing or controlling the direction of movement, therefore it can implement a communication mechanism for the directional information. At two endpoints of this visuo-motor communication system we have implemented neural mechanisms that signal or control the direction by means of the firing patterns of directionally selective populations of neurons [15]. Self-organized learning emerges from the correlated firing of the vision and motor neurons through the means of spike-timing dependent plasticity [18]. A learning step consists of the following sequence of processes: (1) a spontaneous motion is generated by the motor network and maintained for a certain time by a population of neurons coding for the current direction; (2) the eye tracks the moving hand and the motion selective cells from the visual system signal the direction; (3) a cortical (or subcortical) circuit transmits the resulting firing pattern from the visual to the motor control areas; (4) if the arrival of pre-synaptic sensory spikes coincides with a post-synaptic activity of motor neurons coding this particular direction, then an increase of the connection strength results. Finally, a strong selective, one-way connection is established from the vision to the motor assemblies representing the same direction of movement. This facilitates the execution of a movement guided only by visual neural activity [15].

The learning process envisaged above is inspired by the sensorimotor stages that an infant progresses through to develop eye-hand coordination. Within the Piagetian view, the beginnings of imitation appear as the child becomes capable of coordinating hand movements with the incoming visual information, and masters the imitation of hand movements of others. When executing movements during the early motor-babbling period, infants perceive and learn contingencies between the motor activity and the visual image of their actions, hence the simultaneous moving and tracking of an arm can provide the context for the learning process outlined above.

This model supports a particular view on the neurophysiological control of movement. Recent debates on the relation between oculomotor and limb motor control systems advance the hypothesis that eye and hand movements are subject to similar control mechanisms and that gaze (i.e., extraretinal information) provides the signal for target limb motion [2]. We believe that our model provides computational support for the emergence of eye-hand coordination. Specifically, we have obtained an example of "indexing" of a motor set of neurons controlling directionality by another set of neurons which analyze motion direction. Putting it in more general terms, this example supports the thesis that gaze or eye movement neural activity can be "re-used" to control the movement of a limb. From this approach, the conversion of gaze direction into a directionally oriented limb motion do not represent a transformation problem anymore, and become a problem of finding the means for collaboration between active cell assemblies. As support for this thesis, we can cite another example of a developmental process described in [17]. Constructing a system that first learns to foveate a visual target and then "re-use" the saccade map to achieve ballistic reaching provides a compelling example of how motor programs for eye movements (i.e. developed earlier) can provide the computational support for the later acquisition of visually-guided

reaching.

\section{CSRU in developing delayed imita- tion and planning}

Once the basis for immediate action imitation has been established (as described in the previous section), the next developmental stage involves learning complex motor sequences and developing a memory for their representation. From an epigenetic view, deferred imitation marks a progression to the fourth stage of development in human infants, exploiting the functionality of working memory. During this fourth stage, the child also begins to show behavior in which means are clearly differentiated from ends [21]. Infants at nine months of age, are able to search for hidden objects, push aside obstacles and use tools to retrieve distant objects. Our interests reside in modeling the processes that support a parallel development of de- 
layed imitation and intentional, goal-directed behavior.

Essential for articulating a computational approach is that this development occurs gradually and usually by using unplanned forward search with solutions often emerging by accident in the course of trying out several familiar activities [21]. The robot - like the infant - should be able to learn causal relations between commands to its motor region and visual inputs while trying out a sequence of sensory-guided actions (e.g., imitating actions) until eventually a goal is reached. If this succession of actions is externally rewarded, than the system reinforces it and creates a memory representation of the profitable means-effect association. Trough this behavioral adaptation, the agent makes the transition to a stage where preparation and planning of the movements occurs. In infants, planned behavior requires the capacity to organize intentional behavior, defined as: trying to achieve a goal by selecting from among alternative actions, correcting for errors and stopping when the goal is attained [21]. Similar epigenetic approaches have been implemented in several robotic systems, for development of imitative skills [7], intentionality and communication $[6]$.

Current work of our group is focused on implementing the means of asymmetric collaboration and reinforcement learning on a special type of cognitive architecture, to support the emergence of internally initiated, goal-directed sequences of actions.

\subsection{Incremental cognitive architecture}

Considering the nature of the processes that we want to account for, the neurobiologically inspired architecture should involve a sensory (visual or perceptive) input gateway and the frontal lobes' motor and executive areas. We propose a special type of connectionist architecture, which we refer to as an incremental cognitive architecture. The incremental nature relies on the characteristic that some of its components start fully developed and functional, while others will be progressively recruited and became operational by a process of dynamical collaboration, involving synchronization and resonance between the firing patterns of the source and target domains. Among the first modules developed will be those of the visual cortex, proprioceptive area, dorsal premotor and primary motor cortex. The latter may include the supplementary motor area and dorsal prefrontal cortex.

An incremental architecture favors the development of complex behavioral programs in a cascade manner: the activity from the low-level functional modules is applied through cortical connections as a teaching sig-

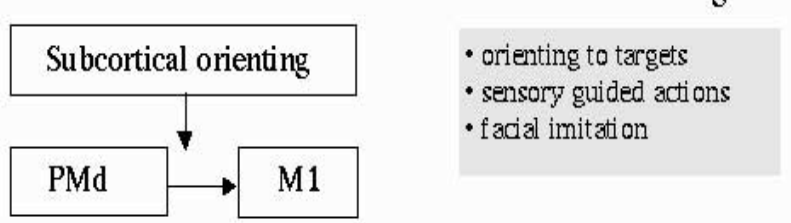

Selective atention Symmetric collaboration

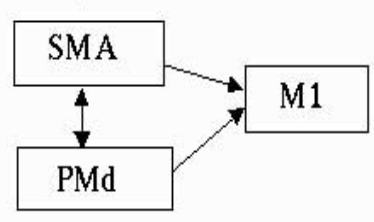

Stage 2

- leaming of motor sequences - internally initi ated actions

- memory for sequences

Reinforcement learning Asymmetric collaboration

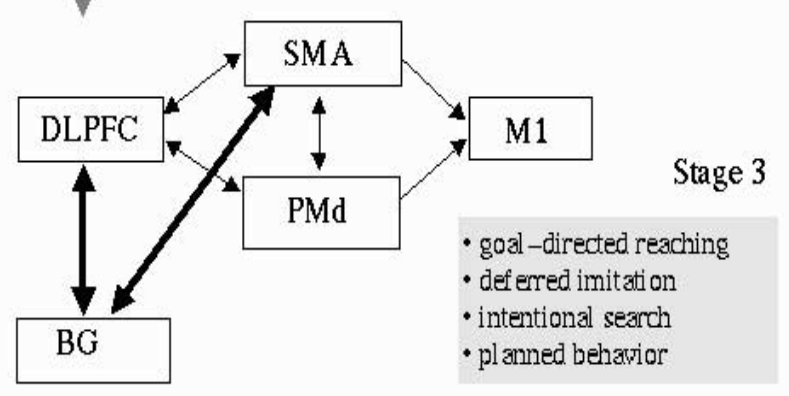

Figure 2: Incremental developmental model. PMd is the dorsal premotor cortex, M1 the primary motor cortex, SMA the supplementary motor area, BG the basal ganglia, and DLPFC the dorsolateral prefrontal cortex. The thick lines designate the cortico-basal ganglia loops via thalamus. Note that the visual areas are not represented here.

nal to the oscillations occurring spontaneously in the higher modules (e.g., SMA, DLPFC). The essential quality of the later developing areas is that the neural populations from here are capable not only of learning the input pattern, but more importantly, are able to abstract a more general and compressed form of behavior, by applying some type of nonlinear combining rule to the patterns they are exposed to. In this process the reciprocal connectivity - from the earlier to the later developed areas and vice versa - plays an essential role. The basal-ganglia through its corticothalamic loops implements the means of reinforcement learning [19].

This architecture is "unfolded" in a developmental model with three stages (Figure 2), each corresponding 
to new behavioral and cognitive acquisitions. A new stage incorporates the achievements of the previous developmental phase and mirrors the recruitment of a new functional module through the means of asymmetric collaboration. Our current and future work focus on implementing this developmental model in a simulated robot and testing the implications of our theory.

\section{Conclusions}

We have presented on-going research on the implementation of a developmental methodology dedicated to the design of intelligent robotic systems. Our approach is based on the cortical software re-use concept, that involve the construction of cognitive functions on a foundation of sensory-motor programs. Two forms of re-use (e.g., neighborhood collaboration and asymmetrically collaborative cell assemblies) have been described at the neural level, giving us, we maintain, the computational means for development of cognitive capabilities. It remains to be seen how further models will confirm or correct the implications of CSRU theory.

\section{References}

[1] J. Demiris, G.M. Hayes, "Imitation as a dual-route process featuring predictive and learning components: a biologically-plausible computational model", Imitation in Animals and Artifacts, K. Dautenhahn, C. Nehaniv (Eds.), MIT Press, 2001.

[2] K.C. Engel, J.H. Anderson, J.F. Soechting, "Similarity in the response of smooth pursuit and manual tracking to a change in the direction of target motion", J. Neurophysiol., 84:3, 1149-1156, 2000.

[3] P. Greenfield, "Language, tool and brain: The ontogeny and phylogeny of hierarchically organized sequential behavior", Behav. Brain Sci., 14:531595, 1991.

[4] D. O. Hebb, The organization of human behavior, New York, Wiley, 1949.

[5] E. Kandel, J. Schwartz, T. Jessell (Eds.), Principles of neural science, McGraw-Hill, 4th Edition, 2000.

[6] H. Kozima, H. Yano, "A robot that learns to communicate with human caregivers", Proc. First Workshop on Epigenetic Robotics, Lund, Sweden, 2001.

[7] Y. Kuniyoshi, A. Nagakubo, L. Berthouze, G. Cheng, "Embodiment, Emergence and Intentionality - A Robotic Point of View", Proc. 6th Symp. on Sohatsu System, Toyama, Japan, 2000.
[8] I. Marian, R. G. Reilly, "SpikeSNNS - a simulator for spike-processing networks", Proc. 12th $D A A A M$ International Symp., Jena, Germany, 2001.

[9] G. Metta, G. Sandini, L. Natale, F. Panerai, "Sensorimotor interaction in a developing robot", Proc. First Workshop on Epigenetic Robotics, Lund, Cognitive Studies, 85, 2001.

[10] J. Piaget, Genetic Epistemology, New York: Columbia University Press, 1970.

[11] R. Port, T. Van Gelder (Eds.), Mind as motion: Explorations in the dynamics of cognition, Cambridge, MA: MIT Press, 1995.

[12] F. Pulvermüller, "Words in the brains language", Behav. Brain Sci., 22:253-336, 1999.

[13] R.G. Reilly, "On the relationship between object assembly and language production: A connectionist simulation of Greenfield's hypothesis", Behav. Brain Sci., in press.

[14] R.G. Reilly, "Collaborative cell assemblies: Building blocks of cortical computation", in Emergent neural computational architectures based on neuroscience, S. Wermter, J. Austin, D. Willshaw (Eds.), Heidelberg, Germany: Springer, 2001.

[15] R.G. Reilly, I. Marian, "A spiking neural model for the visuo-motor imitation", Proc. of neural control of space coding and action production, Lyon, France, 2001.

[17] B. Scassellati, "Building behaviors developmentally: a new formalism", Proc. AAAI Spring Symp. on Integrating Robotics Research, 1998.

[18] S. Song, K.D. Miller, L.F. Abbott, "Competitive Hebbian learning through spike-timing dependent synaptic plasticity", Nature Neurosci., 3:919-926 2000.

[19] R. E. Suri, W. Schultz, "A neural network model with dopamine-like reinforcement signal that learns a spatial delayed response task", Neurosci., 91:3, 871-890, 1999.

[20] J. Weng, J. McClelland, A. Pentland, O. Sporns, I. Stockman, M. Sur, E. Thelen, "Autonomous mental development by robots and animals", Science, 291:5504, 599 - 600, 2000.

[21] P. Willatts, "Development of problem-solving in infancy", in Infant development 